\title{
SECONDARY REPRESENTATIONS FOR INJECTIVE MODULES OVER COMMUTATIVE NOETHERIAN RINGS
}

\author{
by RODNEY Y. SHARP \\ (Received 6th January 1975)
}

\section{Introduction}

There have been several recent accounts of a theory dual to the well-known theory of primary decomposition for modules over a (non-trivial) commutative ring $A$ with identity: see (4), (2) and (9). Here we shall follow Macdonald's terminology from (4) and refer to this dual theory as " secondary representation theory". A secondary representation for an $A$-module $M$ is an expression for $M$ as a finite sum of secondary submodules; just as the zero submodule of a Noetherian $A$-module $X$ has a primary decomposition in $X$, it turns out, as one would expect, that every Artinian $A$-module has a secondary representation.

In the first part of this note, we shall show that, when the ring $A$ is Noetherian, the class of $A$-modules which possess a secondary representation is more extensive than the class of Artinian $A$-modules by proving that every injective $A$-module has a secondary representation. The second part of the note will show how the ideas of the first part are essentially a particular case of a more general functorial argument which gives a procedure for transforming primary decompositions into secondary representations (and vice versa).

We shall use the following notation and terminology. Let $\mathfrak{a}$ be an ideal of $A$ and $N$ be a submodule of the $A$-module $M$. The radical of a will be denoted by $r(\mathfrak{a})$; also, $\left(N:{ }_{M} \mathfrak{a}\right)$ will denote the submodule $\{x \in M: \mathfrak{a} x \subseteq N\}$ of $M$; and $(N: M)$ will denote the ideal $\{a \in A: a M \subseteq N\}$ of $A$, so that, in particular, $(0: M)$ denotes the annihilator of $M$.

As mentioned earlier, we shall follow Macdonald's terminology concerning secondary representation. Thus an $A$-module $S$ is secondary if $S \neq 0$ and, for each $a \in A$, the $A$-endomorphism of $M$ produced by multiplication by $a$ is either surjective or nilpotent. If this is the case, then $\mathfrak{p}=r(0: S)$ is prime, and we say that $S$ is p-secondary. A secondary representation for an $A$-module $N$ is an expression for $N$ as a finite sum of secondary submodules, say

$$
N=S_{1}+S_{2}+\ldots+S_{r},
$$

with $S_{i} \mathfrak{p}_{i}$-secondary (for $i=1, \ldots, r$ ). If such a representation exists, we shall say $N$ is representable. Such a representation is said to be minimal if $\mathfrak{p}_{1}, \mathfrak{p}_{2}, \ldots, \mathfrak{p}_{r}$ are all different and none of the summands $S_{i}$ is redundant. The reader is referred to sections 2, 5 of Macdonald (4) (or to sections 2, 3 of Kirby (2)) 
for proofs of the following facts. Any Artinian $A$-module is representable, and any secondary representation can be refined to a minimal secondary representation. The prime ideals which occur in a minimal secondary representation for a representable $A$-module $N$ (as the radicals of the annihilators of the secondary terms) are uniquely determined by $N$ and are independent of the choice of minimal secondary representation: these prime ideals will be called the attached primes of $N$, and the set which they form will be denoted by $\operatorname{Att}(N)$. $\mathbf{I d}_{X} \cdot$

The identity mapping of an $A$-module $X$ onto itself will be denoted by

\section{Secondary representations for injective modules over a commutative Noetherian} ring

The (non-trivial) commutative ring $A$ with identity will only be assumed to be Noetherian when this is explicitly stated.

Lemma 2.1. Let $\mathfrak{q}$ be a p-primary ideal of $A$, and let $E$ be an injective $A$ module. Then $\left(0:_{E} q\right)$, if non-zero, is $\mathfrak{p}$-secondary.

Proof. Let $a \in A$. If $a \in \mathfrak{p}$, then $a^{n} \in \mathfrak{q}$ for some positive integer $n$, so that $a^{n}$ annihilates $\left(0:_{E} q\right)$. On the other hand, if $a \notin p$, then we can see that

$$
\left(0:_{E} \mathfrak{q}\right)=a\left(0:_{E} \mathfrak{q}\right)
$$

as follows. Let $x \in(0: E q)$. Using - to denote the natural homomorphism from $A$ to $A / \mathfrak{q}$, there is a homomorphism $\phi: A / q \rightarrow E$ for which $\phi(\bar{b})=b x$ for all $b \in A / \mathfrak{q}$.

As the diagram

$$
\begin{aligned}
0 \rightarrow & A / \mathfrak{q} \stackrel{a}{\rightarrow} A / \mathfrak{q} \\
& \downarrow^{\phi} \\
& E
\end{aligned}
$$

has exact row, it can be completed with a homomorphism $\psi: A / \mathfrak{q} \rightarrow E$ which makes the extended diagram commute. Thus $x=\phi(\overline{1})=\psi(a \overline{1})=a \psi(\overline{1})$. Hence (since $\left.\psi(\overline{1}) \in\left(0:_{E} \mathfrak{q}\right)\right)$ we have $\left(0:_{E} \mathfrak{q}\right)=a\left(0:_{E} \mathfrak{q}\right)$, and the result follows. Then

Lemma 2.2. Let $a_{1}, a_{2}, \ldots, a_{n}$ be ideals of $A$ and $E$ an injective $A$-module.

$$
\sum_{i=1}^{n}\left(0:_{E} a_{i}\right)=\left(0:_{E} \bigcap_{i=1}^{n} a_{i}\right)
$$

Proof. Let $x \in\left(0:_{E} \bigcap_{i=1}^{n} a_{i}\right)$. Let $\pi: A \rightarrow A / \bigcap_{i=1}^{n} \mathfrak{a}_{i}$ and, for each $i=1, \ldots, n$, $\pi_{i}: A \rightarrow A / \mathfrak{a}_{i}$, be the natural homomorphisms. There is a monomorphism

$$
\xi: A / \bigcap_{i=1}^{n} \mathfrak{a}_{i} \rightarrow \bigoplus_{i=1}^{n}\left(A / \mathfrak{a}_{i}\right)
$$


for which $\xi(\pi(a))=\left(\pi_{1}(a), \pi_{2}(a), \ldots, \pi_{n}(a)\right)$ for all $a \in A$. Also, there is a homomorphism $\eta: A / \bigcap_{i=1}^{n} a_{i} \rightarrow E$ for which $\eta(\pi(a))=a x$ for all $a \in A$.

As $E$ is injective, we may extend the diagram

$$
0 \rightarrow A \mid \bigcap_{\substack{i=1 \\ \downarrow^{\eta} \\ E}}^{n} a_{i} \stackrel{\xi}{\rightarrow} \bigoplus_{i=1}^{n}\left(A / a_{i}\right)
$$

(which has exact row) by a homomorphism $\zeta: \bigoplus_{i=1}^{n}\left(A / \mathfrak{a}_{i}\right) \rightarrow E$ which makes the extended diagram commute. Now $x=\eta(\pi(1)) \in \operatorname{Im}(\zeta)$, and it is clear that $\operatorname{lm}(\zeta) \subseteq \sum_{i=1}^{n}\left(0:_{E} \mathfrak{a}_{i}\right)$. It follows that $\left(0:_{E} \bigcap_{i=1}^{n} \mathfrak{a}_{i}\right) \subseteq \sum_{i=1}^{n}\left(0:_{E} \mathfrak{a}_{i}\right)$. Since the reverse inclusion is clear, the result follows.

Before we state the main theorem of this section, recall $(10$, p. 46) that an injective $A$-module $E$ is said to be an injective cogenerator of $A$ if, for every $A$-module $X$ and every non-zero $x \in X$, there is a homomorphism $\phi: X \rightarrow E$ such that $\phi(x) \neq 0$.

Theorem 2.3. Assume $A$ is Noetherian, and denote by Ass $(A)$ the set of prime ideals of $A$ which belong to the zero ideal (for primary decomposition). Let $E$ be an injective A-module. Then $E$ has a secondary representation, and Att $(E) \subseteq$ Ass $(A)$.

More precisely, let $0=\mathfrak{q}_{1} \cap \mathfrak{q}_{2} \cap \ldots \cap \mathfrak{q}_{n}$ be a normal primary decomposition for the zero ideal of $A$, with $($ for $i=1, \ldots, n) \mathfrak{q}_{i} a \mathfrak{p}_{i}$-primary ideal. Then

$$
E=\left(0:_{E} \mathfrak{q}_{1}\right)+\left(0:_{E} \mathfrak{q}_{2}\right)+\ldots+\left(0:_{E} \mathfrak{q}_{n}\right),
$$

and $($ for $i=1, \ldots, n)\left(0:_{E} \mathfrak{q}_{i}\right)$ is either zero or $\mathfrak{p}_{i}$-secondary.

Moreover, if $j$ is an integer such that $1 \leqq j \leqq n$, and

$$
J=\{1, \ldots, j-1, j+1, \ldots, n\},
$$

then $E=\sum_{i \in J}\left(0: \mathfrak{q}_{i}\right)$ if and only if $\bigcap_{i \in J} \mathfrak{q}_{i}$ annihilates $E$; consequently, if $E$ is an injective cogenerator of $A$, then $\left(^{*}\right)$ is a minimal secondary representation for $E$, and $\operatorname{Att}(E)=\operatorname{Ass}(A)$.

Proof. 2.1 shows that $\left(0::_{E} \mathfrak{q}_{i}\right)$ is either zero or $\mathfrak{p}_{i}$-secondary, and 2.2 shows that $E=\left(0:_{E} 0\right)=\left(0:_{E} \bigcap_{i=1}^{n} q_{i}\right)=\sum_{i=1}^{n}\left(0:_{E} q_{i}\right)$. The same Lemma also provides the information that, if the integer $j$ satisfies $1 \leqq j \leqq n$, then

$$
\sum_{i \in J}\left(0:_{E} \mathfrak{q}_{i}\right)=\left(0:_{E} \bigcap_{i \in J} \mathfrak{q}_{i}\right)
$$

the latter module is clearly equal to $E$ if and only if $\bigcap_{i \in J} \mathfrak{q}_{i}$ annihilates $E$.

E.M.S. $-20 / 2-K$ 
Now assume $E$ is an injective cogenerator of $A$. To prove the final assertions of the Theorem, it is enough to show that, for each $j=1, \ldots, n$, the ideal $\bigcap_{i \in J} \mathfrak{q}_{i}$ does not annihilate $E$; it is therefore sufficient to show that, if $\mathfrak{b}$ is an arbitrary non-zero ideal of $A$, then $\mathfrak{b}$ does not annihilate $E$.

To this end, let $y$ be a non-zero element of $\mathfrak{b}$. Since $E$ is an injective cogenerator of $A$, there exists a homomorphism $\phi: A \rightarrow E$ such that $\phi(y) \neq 0$. Then $y \phi(1)=\phi(y) \neq 0$, so $\phi(1)$ is an element of $E$ which is not annihilated by $\mathcal{y}$, and so not annihilated by $\mathfrak{b}$. This completes the proof.

In the notation of 2.3 , we can give a more precise description of Att $(E)$ by using the very satisfactory direct decomposition theory for injective modules over a commutative Noetherian ring. Listed below are some of the main facts of this theory; for more details of the theory the reader is referred to (5), (1) and (10). If $M$ is an $A$-module, we shall use $E(M)$ to denote the injective envelope for $M$.

Proposition 2.4. (Matlis; Gabriel.) Assume $A$ is Noetherian.

(i) $A$ direct sum of a family of A-modules is injective if and only if each of the summands is injective. (See (1), §4.)

(ii) There is a bijective correspondence between the prime ideals $\mathfrak{p}$ of $A$ and the isomorphism types of (non-zero) indecomposable injective A-modules given by $\mathfrak{p} \leftrightarrow E(A / \mathrm{p})$. (See Proposition 3.1 of (5).)

(iii) Every injective A-module can be expressed essentially uniquely as a direct sum of indecomposable injective A-modules. (See Theorem 2.5 of (5) and $\S 4$ of (1).)

If follows from this decomposition theory that, if $E$ is an injective module over the Noetherian ring $A$, then there is a family $\left(\mathfrak{p}_{\alpha}\right)_{\alpha \in \Lambda}$ of prime ideals of $A$ for which $E \cong \bigoplus_{\alpha \in \Lambda} E\left(A / \mathfrak{p}_{\alpha}\right)$, and that if $\left(\mathfrak{q}_{\beta}\right)_{\beta \in \Phi}$ is a second family of prime ideals of $A$ for which $E \cong \bigoplus_{\beta \in \Phi} E\left(A / \mathfrak{q}_{\beta}\right)$, then there is a bijection $\gamma: \Lambda \rightarrow \Phi$ such that $\mathfrak{p}_{\alpha}=\mathfrak{q}_{\gamma(\alpha)}$ for all $\alpha \in \Lambda$. The set $\left\{\mathfrak{p}_{\alpha}: \alpha \in \Lambda\right\}$ is thus uniquely determined by $E$; we shall denote this set by $\operatorname{Occ}(E)$, and refer to its members as the prime ideals which occur in the direct decomposition of $E$.

Our next aim is to describe, in the notation of 2.3 , Att $(E)$ in terms of Ass $(A)$ and $\operatorname{Occ}(E)$. To do this, we shall require information about the structure of the indecomposable injective modules over a commutative Noetherian ring.

Lemma 2.5. (Matlis.) Assume $A$ is Noetherian, and $\mathfrak{p}$ is a prime ideal of $A$. 
(i) Every element of $E(A / \mathfrak{p})$ is annihilated by some power of $\mathfrak{p}$. (See Theorem 3.4 (1) of (5).)

(ii) Multiplication by an element $a \in A-\mathfrak{p}$ provides an automorphism of $E(A / \mathrm{p})$. (See Lemma 3.2 (2) of (5).)

(iii) The annihilator of $E(A / \mathfrak{p})$ is $\bigcap_{i=1}^{\infty} \mathfrak{p}^{(i)}$ where (for each $\left.i\right) \mathfrak{p}^{(i)}$ denotes the ith symbolic prime power of p. (See Theorem 3.4 (1) and (2) of (5).) Then

Theorem 2.6. Assume that $A$ is Noetherian and $E$ is an injective A-module.

$$
\text { Att }(E)=\left\{\mathfrak{p}^{\prime} \in \operatorname{Ass}(A): \mathfrak{p}^{\prime} \subseteq \mathfrak{p} \text { for some } \mathfrak{p} \in \operatorname{Occ}(E)\right\} \text {. }
$$

Proof. It was shown in 2.3 that if $0=q_{1} \cap q_{2} \cap \ldots \cap q_{n}$ is a normal primary decomposition for the zero ideal of $A$, with (for $i=1, \ldots, n$ ) $\mathfrak{q}_{i}$ a $\mathfrak{p}_{i}$-primary ideal, then

$$
E=\left(0:_{E} \mathfrak{q}_{1}\right)+\left(0:_{E} \mathfrak{q}_{2}\right)+\ldots+\left(0:_{E} \mathfrak{q}_{n}\right),
$$

and (for $i=1, \ldots, n)\left(0:_{E} \mathfrak{q}_{i}\right)$ is either zero or $\mathfrak{p}_{i}$-secondary. A minimal secondary representation for $E$ can be achieved from $\left(^{*}\right)$ by first removing any zero terms on the right-hand side, and then removing any redundant terms. We shall establish the Theorem by showing $(a)$ that $\left(0:_{E} \mathfrak{q}_{i}\right)=0$ for any $i$ for which $\mathfrak{p}_{i}$ is not contained in any $\mathfrak{p}$ in $\operatorname{Occ}(E)$ and $(b)$ that if $j$ is an integer (with $1 \leqq j \leqq n$ ) for which $\mathfrak{p}_{j}$ is contained in some $p$ belonging to $\operatorname{Occ}(E)$, then $\sum_{i \in J}\left(0:_{E} \mathfrak{q}_{i}\right) \neq E$, where $J=\{1, \ldots, j-1, j+1, \ldots, n\}$, so that $\left(0:{ }_{E} \mathfrak{q}_{j}\right)$ cannot be omitted from $\left(^{*}\right)$ at any stage of the reduction procedure.

(a) Suppose, then, that $i$ is an integer (with $1 \leqq i \leqq n$ ) such that $\mathfrak{p}_{\ell} \nsubseteq \mathfrak{p}$ for all $\mathfrak{p} \in \operatorname{Occ}(E)$. Now if $\left(\mathfrak{p}_{\alpha}\right)_{\alpha \in \Lambda}$ is a family of prime ideals of $A$ for which $E \cong \bigoplus_{\alpha \in \Lambda} E\left(A / \mathfrak{p}_{\alpha}\right)$, then $\left(0:_{E} \mathfrak{q}_{i}\right) \cong \bigoplus_{\alpha \in \Lambda}\left(0:_{E\left(A / p_{\alpha}\right)} \mathfrak{q}_{i}\right)$. Thus, in order to show that $\left(0:_{E} \mathfrak{q}_{i}\right)=0$, it is enough to show that $\left(0:_{E(A / \mathfrak{p})} \mathfrak{q}_{i}\right)=0$ for all $\mathfrak{p} \in \operatorname{Occ}(E)$.

But, for such a $\mathfrak{p}$, we have $\mathfrak{q}_{i} \nsubseteq \mathfrak{p}$ (since $r\left(\mathfrak{q}_{i}\right)=\mathfrak{p}_{i}$ ), so let $a \in \mathfrak{q}_{i}-\mathfrak{p}$. Then multiplication by $a$ on $E(A / \mathfrak{p})$ provides an automorphism of $E(A / \mathfrak{p})$ (by $2.5(\mathrm{ii})$ ), and so $\left(0:_{E(A / \mathfrak{p})} \mathfrak{q}_{i}\right)=0$, as required.

(b) Now suppose $j$ is an integer (with $1 \leqq j \leqq n$ and $J$ defined as before) for which $\mathfrak{p}_{j} \subseteq \mathfrak{p}$ for some $\mathfrak{p} \in \operatorname{Occ}(E)$. We shall show that $\sum_{i \in J}\left(0 i_{E} \mathfrak{q}_{i}\right) \neq E$. Suppose this is not the case; then, by $2.3, E$ is annihilated by $\mathfrak{r}_{j}=\bigcap_{i \in J} \mathfrak{q}_{i}$, and therefore $E(A / \mathfrak{p})$ is annihilated by $\mathfrak{r}_{j}$. Hence, by $2.5\left(\right.$ iii), $\mathfrak{r}_{j} \subseteq \bigcap_{k=1}^{\infty} \mathfrak{p}^{(k)}$. Now $\mathbf{r}_{j} \nsubseteq \mathfrak{q}_{j}$; let $a \in \mathfrak{r}_{j}-\mathfrak{q}_{j}$. Then $a \in \bigcap_{k=1}^{\infty} \mathfrak{p}^{(k)}$, which is the kernel of the natural ring homomorphism from $A$ to $A_{p}$. Hence there exists $s \in A-p$ such that $s a=0 \in \mathfrak{q}_{j}$. Since $a \in A-\mathfrak{q}_{j}$ and (as $\mathfrak{p}_{j} \subseteq \mathfrak{p}$ ) $s \in A-\mathfrak{p}_{j}$, this is in contradiction to the fact that $\mathfrak{q}_{j}$ is $\mathfrak{p}_{j}$-primary.

This completes the proof. 


\section{More general arguments of a functorial nature}

In this section (in which we shall again assume only that $A$ is a (non-trivial) commutative ring with identity) we shall show that the argument which led to Theorem 2.3 is essentially a particular case of a more general functorial argument. Let $\mathscr{C}(A)$ denote the category of all $A$-modules and $A$-homomorphisms. Let $E$ be an injective $A$-module; then there is a natural isomorphism between $E$ and $\operatorname{Hom}_{A}(A, E)$ under which the submodule $\left(0:_{E} \mathfrak{q}\right)$ of $E$ (for an ideal $\mathfrak{q}$ ) corresponds to the image of the homomorphism

$$
\operatorname{Hom}_{A}(A / \mathrm{q}, E) \rightarrow \operatorname{Hom}_{A}(A, E)
$$

induced by the natural epimorphism $A \rightarrow A / \mathfrak{q}$. Now $\operatorname{Hom}_{A}(, E)$ is a contravariant exact additive $A$-linear $(7, \S 3.7)$ functor from $\mathscr{C}(A)$ to itself. In this section, $T$ will denote a contravariant exact additive $A$-linear functor from $\mathscr{C}(A)$ to itself; we shall indicate how $T$ transforms primary decompositions into secondary representations. As it should become clear that the first part of Section 2 is essentially a special case of the argument of this section, we shall only give indications of proof in this section: the interested reader should be able to fill in the details for himself.

3.1. The theory of primary decomposition for $A$-modules is so well documented that it is only necessary to specify the terminology which is to be used. We shall assign to the terms primary submodule, $p$-primary submodule, and (normal) primary decomposition in an $A$-module $M$ (of a submodule of $M$ ) the same meanings as they have in sections 2.8 and 2.9 of Northcott (8). When we say that an $A$-module $M$ has (p-)primary (resp. decomposable) zero submodule, then the qualification " in $M$ " is to be understood.

Let $M$ be an $A$-module. It is clear that the study of primary decompositions in $M$ of a submodule $N$ of $M$ is effectively the same as the study of primary decompositions of the zero submodule of $M / N$. Note that $M$ has primary zero submodule if and only if $M \neq 0$ and, for all $a \in A$, the endomorphism of $M$ produced by multiplication by $a$ is either injective or nilpotent.

If $M$ has decomposable zero submodule, the prime ideals of $A$ which belong (8, p. 106) to the zero submodule of $M$ will be called the associated primes of $M$; the set which they form will be denoted by Ass $(M)$.

3.2. Suppose $Q$ is an $A$-module having $p$-primary zero submodule. For $a \in A$ and each integer $i>0$, we have $T\left(a^{i} \cdot \operatorname{Id}_{Q}\right)=a^{i} \cdot T\left(\operatorname{Id}_{Q}\right)=a^{i} \cdot \operatorname{Id}_{T(Q)}$. Now if $a \in \mathfrak{p}$, there is an integer $n>0$ for which $a^{n}$. Id $\mathrm{d}_{Q}$ is the zero endomorphism of $Q$, so that $a^{n} \cdot \operatorname{Id}_{T(Q)}$ is the zero endomorphism of $T(Q)$. On the other hand, if $a \notin \mathfrak{p}$, then $a$. Id $\mathbf{d}_{Q}$ is monomorphic, so that the properties of $T$ ensure that $a \cdot \mathrm{Id}_{T(Q)}$ is epimorphic. Thus $T(Q)$, if non-zero, is $p$-secondary.

In a similar manner, one can show that, if $S$ is a $p$-secondary $A$-module, then $T(S)$ is either zero or has $p$-primary zero submodule.

3.3. Suppose $M$ is an $A$-module having decomposable zero submodule; let $0=Q_{1} \cap Q_{2} \cap \ldots \cap Q_{n}$ be a normal primary decomposition for the zero 
submodule of $M$, with (for $i=1, \ldots, n$ ) $Q_{i}$ a $\mathfrak{p}_{i}$-primary submodule of $M$. For each $i=1, \ldots, n$, let $\pi_{i}: M \rightarrow M / Q_{i}$ be the natural epimorphism;

$$
T\left(\pi_{i}\right): T\left(M / Q_{i}\right) \rightarrow T(M)
$$

will be monomorphic, and since $M / Q_{i}$ has $\mathfrak{p}_{i}$-primary zero submodule, 3.2 shows that $S_{i}=T\left(\pi_{i}\right) T\left(M / Q_{i}\right)$ is either zero or $p_{i}$-secondary.

Next, let $X=\bigoplus_{i=1}^{n}\left(M / Q_{i}\right)$ and let $f: M \rightarrow X$ be the homomorphism for which $f(m)=\left(\pi_{1}(m), \pi_{2}(m), \ldots, \pi_{n}(m)\right)$ for all $m \in M$. Since $f$ is monomorphic, $T(f): T(X) \rightarrow T(M)$ must be epimorphic. It is easy to see from this that

$$
T(M)=S_{1}+S_{2}+\ldots+S_{n}
$$

this argument therefore shows that, whenever $M$ is an $A$-module having decomposable zero submodule, then $T(M)$ is representable with

$$
\text { Att }(T(M)) \subseteq \text { Ass }(M) \text {. }
$$

3.4. Here, we use the same notation as in 3.3. Suppose $j$ is an integer such that $1 \leqq j \leqq n$, define $j$ as before, and let $Y_{j}=\bigoplus_{i \in J}\left(M / Q_{i}\right)$. There is a natural homomorphism $h_{j}: M \rightarrow Y_{j}$ which has kernel $K_{j}=\bigcap_{i \in J} Q_{i}$. It is straightforward to show that $T\left(h_{j}\right): T\left(Y_{j}\right) \rightarrow T(M)$ has $\sum_{i \in J} S_{i}$ for its image. Applying $T$ to the exact sequence

$$
0 \rightarrow K_{j} \rightarrow M^{h_{j}} \rightarrow Y_{j}
$$

now shows that $T(M)=\sum_{i \in J} S_{i}$ if and only if $T\left(K_{j}\right)=0$. Note that, as we began with a normal primary decomposition of the zero submodule of $M$, each $K_{j}(j=1, \ldots, n)$ is non-zero.

Now suppose that $T$ has the additional property of being faithful, i.e. whenever $g: Z \rightarrow W$ is a non-zero homomorphism of $A$-modules, then

$$
T(g): T(W) \rightarrow T(Z)
$$

is non-zero also, or, equivalently, whenever $V$ is a non-zero $A$-module, then $T(V) \neq 0$ also. (An example of such a functor would be $\operatorname{Hom}_{A}\left(, E^{\prime}\right.$ ), where $E^{\prime}$ is an injective cogenerator of $A$.) It follows easily from the preceding paragraph and 3.3 that

$$
T(M)=S_{1}+S_{2}+\ldots+S_{n}
$$

is actually a minimal secondary representation, so that

$$
\text { Att }(T(M))=\left\{\mathfrak{p}_{1}, \mathfrak{p}_{2}, \ldots, \mathfrak{p}_{n}\right\} \text {. }
$$

Thus, when $T$ is faithful and $M$ is an $A$-module having decomposable zero submodule, then not only is $T(M)$ representable, but also

$$
\text { Att }(T(M))=\text { Ass }(M) \text {. }
$$

3.5. An application. Let $R$ be a commutative Noetherian local ring (with identity) and $E$ be the injective envelope of the residue field of $R$. It is well 
known that, whenever $N$ is a finitely generated $R$-module, then $\operatorname{Hom}_{R}(N, E)$ is an Artinian $R$-module (10, $\S 3.4$ and Theorem 4.30) and so is certainly representable. Since $N$, being Noetherian, has decomposable zero submodule, it is natural to look for connections between $\operatorname{Ass}(N)$ and $\operatorname{Att}\left(\operatorname{Hom}_{R}(N, E)\right)$. In the special case in which $R$ is complete, one can use Matlis's duality in conjunction with alternative characterisations of Ass $(N)$ and $\operatorname{Att}\left(\operatorname{Hom}_{R}(N, E)\right)$ to show that these two sets are equal: see 2.3 of (3). However, 3.4 above deals with the general, not necessarily complete, case, because $E$ is an injective cogenerator of $R$ : it follows not only that $\operatorname{Att}\left(\operatorname{Hom}_{R}(N, E)\right)=\operatorname{Ass}(N)$, but also that a normal primary decomposition of the zero submodule of $N$ gives rise in a natural way to a secondary representation of $\operatorname{Hom}_{R}(N, E)$.

3.6. Concluding remarks. In 3.1-3.4, we were principally concerned with the transformation by our contravariant functor $T$ of primary decompositions into secondary representations. There are three parallel families of results, one concerning the transformation by $T$ of secondary representations into primary decompositions, and the others concerning the manner in which a covariant exact additive $A$-linear functor transforms primary decompositions into primary decompositions, and secondary representations into secondary representations.

However, in connection with these parallel results, it should be pointed out that the ideas of Moore (6) enable us to interpret a secondary representation for a module $X$ in $\mathscr{C}(A)$ in terms of a primary decomposition for the null subobject of $X$ when $X$ is considered as an object in $\mathscr{C}(A)^{*}$, the dual category of $\mathscr{C}(A)$. Indeed, Moore's work and the essentially categorical flavour of the above arguments in Section 3 suggest that the most natural setting for studying the various parallel families of results might be provided by Abelian categories over $A$, for the situations of all the results mentioned above are all special cases of the following more general situation: $\mathscr{B}$ and $\mathscr{D}$ are both Abelian categories over $A$, a primary decomposition of the null subobject of an object $Y$ of $\mathscr{B}$ is given, and one considers the effect of a covariant exact additive $A$-linear functor $V: \mathscr{B} \rightarrow \mathscr{D}$. However, these ideas are superfluous to the main aim of this note, and so we shall not discuss them further here.

\section{REFERENCES}

(1) P. GabrIEL, Objets injectifs dans les catégories abéliennes, Sém. DubreilPisot. Fas. 12, Exposé 17 (1958-9).

(2) D. KIRBY, Coprimary decomposition of Artinian modules, J. London Math. Soc. 6 (1973), 571-576.

(3) I. G. MACDONALD and R. Y. Sharp, An elementary proof of the non-vanishing of certain local cohomology modules, Quart. J. Math. Oxford (2), 23 (1972), 197-204.

(4) I. G. MACDONALD, Secondary representation of modules over a commutative ring, Symposia Mathematica 11 (1973), 23-43. 
SECONDARY REPRESENTATIONS FOR INJECTIVE MODULES 151

(5) E. Matuis, Injective modules over Noetherian rings, Pacific J. Math. 8 (1958), 511-528.

(6) D. J. Moore, Primary and coprimary decompositions, Proc. Edinburgh Math. Soc. 18 (1973), 251-264.

(7) D. G. NoRTHCOTr, An introduction to homological algebra (Cambridge University Press, 1960).

(8) D. G. NoRThCotT, Lessons on rings, modules and multiplicities (Cambridge University Press, 1968).

(9) D. G. NorthCotr, Generalized Koszul complexes and Artinian modules, Quart. J. Math. Oxford (2), 23 (1972), 289-297.

(10) D. W. Sharpe and P. VÁmos, Injective modules (Cambridge tracts in mathematics and mathematical physics No. 62, Cambridge University Press, 1972).

Department of Pure Mathematics

UNIVERSITY OF SHEFFIELD

SHEFFIELD S3 7RH 